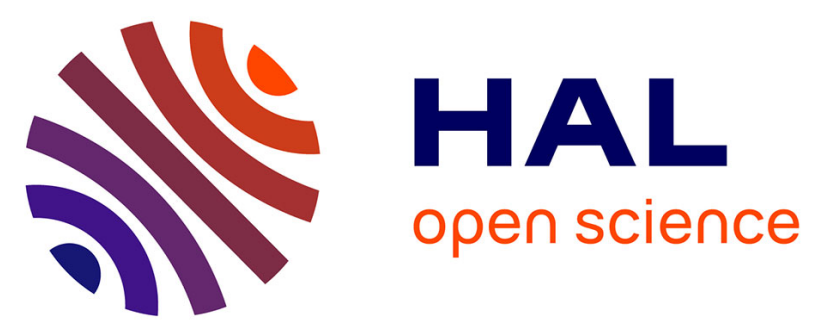

\title{
Le programme ANR Pont-Euxin: bilan des campagnes 2012 à Apollonia du Pont (Sozopol, dpt. de Bourgas, Bulgarie) et Orgamè / Argamum (Jurilovca, dpt. de Tulcea, Roumanie)
}

Alexandre Baralis, Vasilica Lungu, Krastina Panayotova, Thierry Blanco, Guenaelle Bony, Anthony Comfort, Laurent Claquin, R. Delfieu, Alexandra Dolea, Pierre Dupont, et al.

\section{- To cite this version:}

Alexandre Baralis, Vasilica Lungu, Krastina Panayotova, Thierry Blanco, Guenaelle Bony, et al.. Le programme ANR Pont-Euxin: bilan des campagnes 2012 à Apollonia du Pont (Sozopol, dpt. de Bourgas, Bulgarie) et Orgamè / Argamum (Jurilovca, dpt. de Tulcea, Roumanie). Dialogues d'histoire ancienne, 2012, 38 (2), pp.165-187. 10.3406/dha.2012.3447 . halshs-01297512

\section{HAL Id: halshs-01297512 \\ https://shs.hal.science/halshs-01297512}

Submitted on 4 Apr 2016

HAL is a multi-disciplinary open access archive for the deposit and dissemination of scientific research documents, whether they are published or not. The documents may come from teaching and research institutions in France or abroad, or from public or private research centers.
L'archive ouverte pluridisciplinaire HAL, est destinée au dépôt et à la diffusion de documents scientifiques de niveau recherche, publiés ou non, émanant des établissements d'enseignement et de recherche français ou étrangers, des laboratoires publics ou privés. 
Le programme ANR Pont-Euxin : bilan des campagnes 2012 à Apollonia du Pont (Sozopol, dpt. de Bourgas, Bulgarie) et Orgamè / Argamum (Jurilovca, dpt. de Tulcea, Roumanie)

Au terme de sa troisième année d'exercice, le programme Pont-Euxin a obtenu tout à la fois une prolongation de douze mois accordée par l'Agence Nationale de la Recherche, ainsi qu'un élargissement de ses supports financiers. Ses activités en Bulgarie intègrent désormais la nouvelle Mission archéologique française à Apollonia du Pont (dir. A. Baralis), financée par le Ministère des Affaires Étrangères. Les travaux de nos équipes ont bénéficié sur ce point du dynamisme de l'Ambassade de France et de l'Institut culturel français de Sofia, fortement engagés tous deux aux côtés des équipes archéologiques œuvrant sur le terrain. Sur le plan scientifique, 2012 se présente comme une année de transition, marquée par l'achèvement du programme initial de fouilles à Orgamè (Roumanie), ainsi que par le lancement des analyses transversales, prélude à la publication monographique des résultats acquis sur le terrain depuis 2010. Cette étape du travail répond à la livraison de nombreux résultats d'analyses, tant dans le domaine de l'archéométrie que dans celui des disciplines paléoenvironnementales (sédimentologie, palynologie, macrobiologie...).

\section{Orgamè / Argamum, Jurilovca, dpt. de Tulcea, Roumanie}

Succédant à la campagne de carottage réalisée en mars 20 ir le long des rives sud de la Péninsule de Dolojman, ainsi que sur l'île voisine de Bisericuța, les analyses sédimentologiques et palynologiques des échantillons menées en laboratoire (palynologie : Ecolab, I. Rossignol, dir. D. Kaniewski ; sédimentologie : Cerege, G. Bony, dir. Ch. Morhange) ont permis de jeter un éclairage précieux et inattendu sur l'évolution des paysages aux alentours d'Orgamè. La transition entre un golfe marin ouvert sur la haute mer et la formation d'un espace fortement influencé par les alluvions du Danube s'opère à une date relativement haute, calée entre deux datations ${ }^{14} \mathrm{C}(\mathrm{ca} .3500-3350 \mathrm{et}$ 2350-2200 av. J.-C., carottages $\mathrm{O}_{1}$ et $\mathrm{O}_{2}$ ) qui permettent de situer cette étape majeure durant l'Âge du Bronze Ancien. En regard, le développement de la présence grecque sur le cap Dolojman et le long des rives du golfe de Baia prend place au sein d'un milieu déjà lagunaire. Ce dernier disposait de qualités certaines, en particulier sur le plan halieutique, offrant un écho direct aux témoignages épigraphiques plus tardifs qui détaillent l'activité des habitants d'Istros à l'embouchure du Danube et potentiellement plus au sud, bien que cette dernière information repose sur un fragment lacunaire de l'orothésie de Laberius Maximus. La fermeture de l'actuelle lagune du Razelm intervient pour sa 
part durant les premiers siècles de notre ère. Une date (ca 380-600 apr. J.-C., carottage OI), obtenue au début d'un faciès marécageux, nous sert ici de guide et apporte un précieux terminus ante quem. Il semble dès lors que la dernière période d'expansion de la cité d'Orgamè/Argamum ait eu pour décor un environnement assez proche de celui que nous lui connaissons aujourd'hui. Ce schéma inattendu s'inscrit pourtant en parfaite cohérence avec les diagrammes polliniques où l'on observe une sensible expansion des genres aquaphiles de type Sparganium / Typha dès le VI ${ }^{\mathrm{e}}$ s. av. J.-C. Leur présence le long du littoral est également confirmée par les analyses macrobiologiques (I. Slavova, dir. E. Marinova-Wolff) conduites sur les sédiments provenant de l'établissement grec de Caraburun-Atchik Suhat, ainsi que des fosses de l'habitat gète de Zimbru, fouillées respectivement en 201 i et en 2010 dans le cadre de ce programme. Plusieurs taxons carbonisés révèlent leur utilisation entre le $\mathrm{VI}^{\mathrm{e}}$ et le $\mathrm{IV}^{\mathrm{e}} \mathrm{s}$. av. J.-C. dans les édifices de ces deux sites contemporains d'Orgamè. L'ensemble de ces observations permet donc de resituer la description que nous livre Polybe d'une longue flèche sableuse, disposée au sud de l'embouchure du Danube et connue alors sous le terme de $\sigma \tau \dot{\eta} \theta \eta$. Cette attestation d'un espace littoral déjà déconnecté de la haute mer se retrouve également au siècle suivant chez Strabon qui mentionne à plusieurs reprises les « marais » de la Scythie mineure. En ce sens, il n'est pas inintéressant de rappeler que l'orothésie du territoire d'Istros de Laberius Maximus, désigne vers ıoo apr. J.-C. le golfe d'Halmyris, au nom en lui-même révélateur, sous le terme de « laccus » (sic).

Cette évolution des zones littorales s'accompagne par ailleurs d'une métamorphose des paysages régionaux dont les diagrammes polliniques et les analyses numériques dressent les grandes phases. Ils soulignent en ce sens l'existence au début du II ${ }^{\mathrm{e}}$ millénaire avant notre ère d'un couvert forestier encore important dans lequel prédominent le pin et le chêne décidu. La transition entre l'Âge du Bronze et le Premier Âge du Fer accompagne un changement des conditions climatiques, jusqu'alors humides, vers un ensemble plus aride, à un moment où la carte archéologique enregistre de son côté une densité inédite d'habitats. Ce phénomène engage alors une décroissance du chêne, favorisant en retour l'expansion du pin et des graminées. Le couvert forestier est alors remplacé le long du littoral par une steppe dominée par les Chenopodiaceae. Une augmentation de la fréquence des espèces aquatiques est pourtant visible dans les quelques siècles qui précèdent le début de notre ère, indiquant la présence d'eau douce dans les secteurs proches d'Orgamè. Parallèlement, d'importantes variations climatiques sont observées durant l'ensemble de l'Antiquité. La steppe reste dominante mais une dynamique arborée persiste. Ce processus s'interrompt au cours de l'Antiquité tardive pour laisser place à un fort recul qui affecte alors toutes les espèces. Zones de 
steppe et espaces cultivés connaissent durant cette période une brutale expansion qui accompagne une multiplication des établissements recensés dans le triangle BabadagBeidaud-Panduru, tandis que se met en place sur le territoire proche d'Orgamè un réseau dense d'édifices ruraux. Cette variabilité climatique se poursuit plus tard durant la période médiévale, laquelle engage une dynamique plus rapide entre écosystèmes steppiques et arborés, générant une alternance de ces deux types de milieux sur de courtes périodes. L'essor, durant les IX'/ $\mathrm{X}^{\mathrm{c}}$ s. apr.J.-C., des habitats relevant de la culture Dridu, s'accompagne en ce sens d'un nouveau recul du pin, certes limité, et dans une moindre mesure du chêne. À l'inverse, la fin de la période médiévale et le début de l'époque ottomane coïncident avec une récupération de la couverture forestière. L'analyse enfin des pluies polliniques actuelles permet de définir deux types d'écosystèmes sur le territoire d'Orgamè, un milieu steppique dominée par les Chenopodiaceae et les Artemisia et un second plus humide de type marais où les Cyperaceae et les Sparganium/Typha sont majoritaires dans les reliefs les plus bas du territoire?

Au-delà de ce schéma global qui dresse dans une perspective diachronique les grandes lignes de l'évolution de l'environnement proche d'Orgamè, il est désormais possible, grâce aux échantillons macrobiologiques issus des fouilles archéologiques, de changer tout à la fois d'échelle spatiale et chronologique afin d'approcher la composition des micro-paysages entourant les sites archéologiques, tout en évaluant l'impact de ces derniers sur les écosystèmes régionaux. C'est notamment le cas de l'établissement gète de Zimbru dont le matériel récolté dans le remplissage des fosses à déchets apporte un éclairage inédit sur l'économie d'un habitat indigène contemporain de la colonie grecque (fig. I et 2). Les résultats des analyses réservent à ce propos quelques surprises. Tout d'abord, la ventilation des espèces arboricoles utilisées à Zimbru pour la construction ou la combustion ne reproduit pas le faciès du couvert forestier contemporain. Alors que le pin prédomine dans les assemblages polliniques, chêne et charme sont ici largement surreprésentés, révélant un processus de sélection qui impacte davantage certaines espèces. De même, l'éventail des essences s'avère relativement large (érable, frêne, prunus...) et comprend des espèces disposées à distance du littoral. Les habitants de Zimbru n'adoptent donc pas le caractère opportuniste constaté sur de nombreux autres sites. Tout aussi révélatrice, l'identité des espèces cultivées permet de fixer les paysages autour de cet établissement. Les cultures céréalières prédominent

M. Petrescu, Dobrogea şi Delta Dunarii: conservarea florei şi habitatelor, Biblioteca Istro-Pontica II, Tulcea, 2007, 355 p. ; I. Rossignol, D. Kaniewski, E. Van Campo, M. Petrescu, A. Baralis, « A modern pollen rain study from the Black Sea coast of Romania », Review of Palaeobotany and Palynology 174 (2012), p. 39-47. 


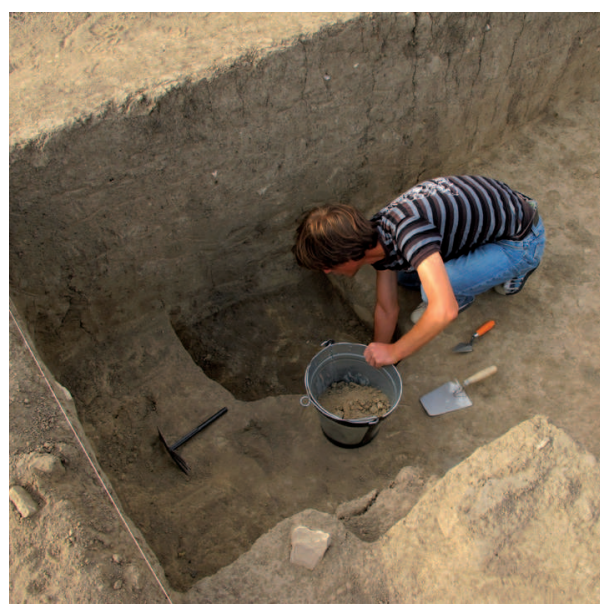

Fig. 1. Fouille du site de Zimbru. Cl. V. Lungu.

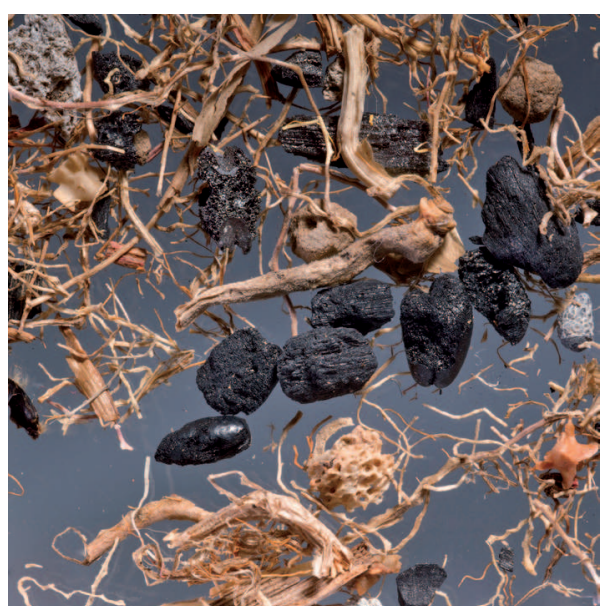

Fig. 2. Matériel macrobiologique de la fosse S. VII. Cl. Ch. Durand (CCJ).

les assemblages ( $84 \%$ de l'ensemble du matériel), mais contre toute attente, le blé tendre (Tr. aestivum / durum L.), caractéristique des territoires des colonies grecques pontiques, est ici préféré à l'engrain et l'amidonnier (Tr. monococcum / dicoccum), pourtant plus rustiques. Il entre également en association avec le millet et l'orge. Zimbru partage donc sur ce point un faciès particulièrement proche avec celui identifié en 2ori sur l'établissement grec littoral de Caraburun-Atchik Suhat. Les légumineuses pour leur part, cultivées à proximité du village, s'articulent autour du pois (Pisum sativum L.), des lentilles (Lens culinaris) et de la vesce amère (Vicia ervilia L.). Les fruits enfin proviennent tout à la fois d'activités de cueillette, notamment pour le sureau noir (Sambucus sp. L.), le merisier (Prunus avium L.) et la cornouille (Cornus mas L.), et d'espèces cultivées comme dans le cas très particulier de la vigne (Vitis vinifera L.) laissant ouverte la possibilité d'une production viticole dans cet habitat gète disposé aux limites du territoire agricole de la cité. En regard, l'assemblage provenant de l'établissement grec contemporain de Caraburun-Atchik Suhat s'avère relativement plus pauvre. Seule la lentille a été identifiée parmi les légumineuses, sans qu'il soit possible d'exclure tout biais dans l'échantillonnage. On note de même sur ce dernier site l'emploi manifeste de phragmites dans les constructions, révélant ici en contexte grec l'existence de dispositifs de couverture similaires à celui reconnu sur les villages gètes littoraux. Enfin, le prélèvement de la masse ligneuse s'avère tout aussi sélectif et privilégie également le chêne. Toutefois, le profil des espèces secondaires, articulé autour du noisetier et du charme, diffère ici nettement de celui identifié à Zimbru. 
Si nous ne disposons pas d'un matériel équivalent pour le site d'Orgamè, son territoire agricole, disposé entre le village moderne de Jurilovca et le cap Dolojman, s'avère désormais mieux connu grâce aux campagnes de prospections systématiques conduites en 2010 et 201 (A. Baralis, V. Lungu, P. Lebouteiller). Ces travaux ont révélé l'absence d'édifices isolés sur une très longue période, depuis la fondation de l'établissement grec jusqu'à la mise en place d'un paysage rural densément occupé durant la période tardoromaine / protobyzantine. Toutefois, en raison de la rotation moderne des cultures et de l'absence de labours près de la cité, seuls les secteurs méridionaux avaient fait l'objet jusqu'à présent d'une couverture complète. En regard, le système d'occupation au nord de la péninsule, tout comme les espaces proches de la cité, n'avaient pas bénéficié de la même attention, malgré les données, nombreuses, accumulées depuis le début des fouilles régulières en 1988 de la nécropole par V. Lungu. Afin de combler cette lacune, une dernière campagne de prospections a donc été organisée du 8 au is avril 2012 en profitant de la fenêtre offerte par l'incendie qui a parcouru l'O. de la péninsule durant l'automne 20II. Près de 40,75 ha ont été couverts, lesquels s'ajoutent aux I30 ha parcourus précédemment, portant la surface totale étudiée à 170,75 ha. Ces travaux ont permis d'ajouter ainsi 5 édifices ruraux protobyzantins à la carte archéologique, lesquels complètent les I2 autres précédemment reconnus. Au N. de la basilique extra-muros n ${ }^{\circ}$ 4, l'habitat se répartit durant la fin de l'Antiquité de part et d'autre de l'axe de circulation qui longe le nord de la péninsule en exploitant ici les riches terres noires créées par l'écoulement des eaux le long du fond de vallée qui débouche au sud de la péninsule, à proximité de la cité. Dans la zone adjacente à l'espace urbain, ils se juxtaposent aux ensembles tumulaires hellénistiques, en particulier le long de la route médiane qui rejoint la « Petite Porte », illustrant la transition déjà observée sur cet espace entre les secteurs funéraires $\left(\mathrm{IV}^{\mathrm{e}}-\mathrm{III}^{\mathrm{e}} \mathrm{s}\right.$. av. J.-C.), et les diverses zones d'habitation qui se mettent en place durant la période tardo-romaine. Autre acquis, les limites du faubourg extra-muros semblent devoir être élargies, car la répartition des tessons et des éléments architectoniques au sol laissent entrevoir l'extension de ce vaste quartier le long du littoral, au $S$. de la péninsule, sur près de $0,6 \mathrm{~km}$. Suivant l'actuel chemin de terre qui conduit au site, il se développe sous la forme d'une large bande qui dépasse le cap qui marque la limite occidentale de la zone portuaire pour atteindre une étroite baie dont la fonction reste ouverte. La question de la relation chronologique qu'entretiennent ces deux phénomènes, à savoir la dilatation de l'espace urbain au-delà des remparts et la mise en place d'un réseau d'occupation relativement dense sur la péninsule, devra à l'avenir être précisée.

Si les prospections géomagnétiques sur l'espace urbain, programmées initialement en 20I2, n'ont pu encore être réalisées pour des raisons de calendrier, les campagnes 
archéologiques de juin et d'août ont permis en revanche d'achever la fouille du secteur funéraire TIV.2010 (V. Lungu), ainsi que des deux édifices ruraux « Casa Romana » I et 2 (A. Dolea, A. Muşat, M. Streinu). Le secteur TIV.20Io disposé immédiatement au sud de « Casa romana » I, constitue en effet jusqu'à présent l'ensemble funéraire le plus éloigné de la cité et marque en l'état la frontière occidentale de la nécropole. Les travaux de 201 a avaient mis au jour un premier tumulus de 3,5 $\mathrm{m}$ de diamètre, daté de la seconde moitié du IV ${ }^{\mathrm{c}} s$. av. J.-C. La campagne d'août 2012 a révélé la présence immédiatement au nord d'un long mur large de $0,80 \mathrm{~m}$, suivi sur plus de $20 \mathrm{~m}$. Conservé sur o,I2 $\mathrm{m}$ de hauteur, il s'organise autour d'un parement externe réalisé en moellons de calcaire disposés en carreaux selon un appareil pseudo-isodome, lequel s'appuie sur un blocage épais. Cette construction, particulièrement soignée, s'avère inédite à Orgamè et témoigne tout à la fois du statut de la sépulture qu'elle protège ainsi que de l'importance manifeste de l'axe de circulation adjacent. Elle trahit par ailleurs la sensibilité de cette colonie, au profil au demeurant très conservateur dans ses rites funéraires, face aux modes et aux innovations architecturales qui traversent alors le monde grec. Plus au nord, les travaux engagés ont dégagé l'intégralité de « Casa Romana 》 I, dévoilant l'aménagement interne de cette construction unicamérale de $6,33 \times 7,75 \mathrm{~m}$, orientée $I^{\circ}$ nord. Au sol, un foyer, matérialisé par une zone rubéfiée disposée près du mur oriental, apparaît dépourvu de tout dispositif de protection pérenne. De même, une fosse, profonde de o,60 m, occupait approximativement le centre de cet espace. Son remplissage, intégralement prélevé, est actuellement en cours d'analyse. Enfin, le long de la façade orientale, deux trous de poteaux rappellent la présence d'un appentis. Plus énigmatiques, trois autres trous, renforcés par un blocage de moellons, ont été identifiés au sein de l'édifice, sans que leur disposition ne respecte l'orientation actuelle de l'édifice. Ils posent en retour la question de l'existence éventuelle d'une première étape dans la construction de bâtiment, réalisée en matériaux périssables. Cette hypothèse, tentante à première vue, se heurte pourtant à la stratigraphie générale du site, tout comme à la répartition des tessons dans son remplissage. Indépendamment de cette question, l'étude du matériel céramique, actuellement conduite par T. Blanco, A. Dolea, A. Muşat et M. S. Streinu, permettra à terme de préciser la chronologie de cet ensemble daté pour l'instant des $V^{e}-V^{e}$ s. apr. J.-C. Plus riche en matériel, « Casa Romana » I s'avère distante de seulement $0,2 \mathrm{~km}$. Elle adopte également des dimensions relativement proches et une orientation strictement parallèle. Dans son angle interne S.O., deux murs isolent un modeste réduit. Deux zones semblent avoir joué un rôle particulier. Une première, présente à l'E. de la pièce secondaire, a livré de nombreux outils, tandis qu'un foyer, identifié au centre, était accompagné par une quantité notable de vases de cuisine et de céramique 


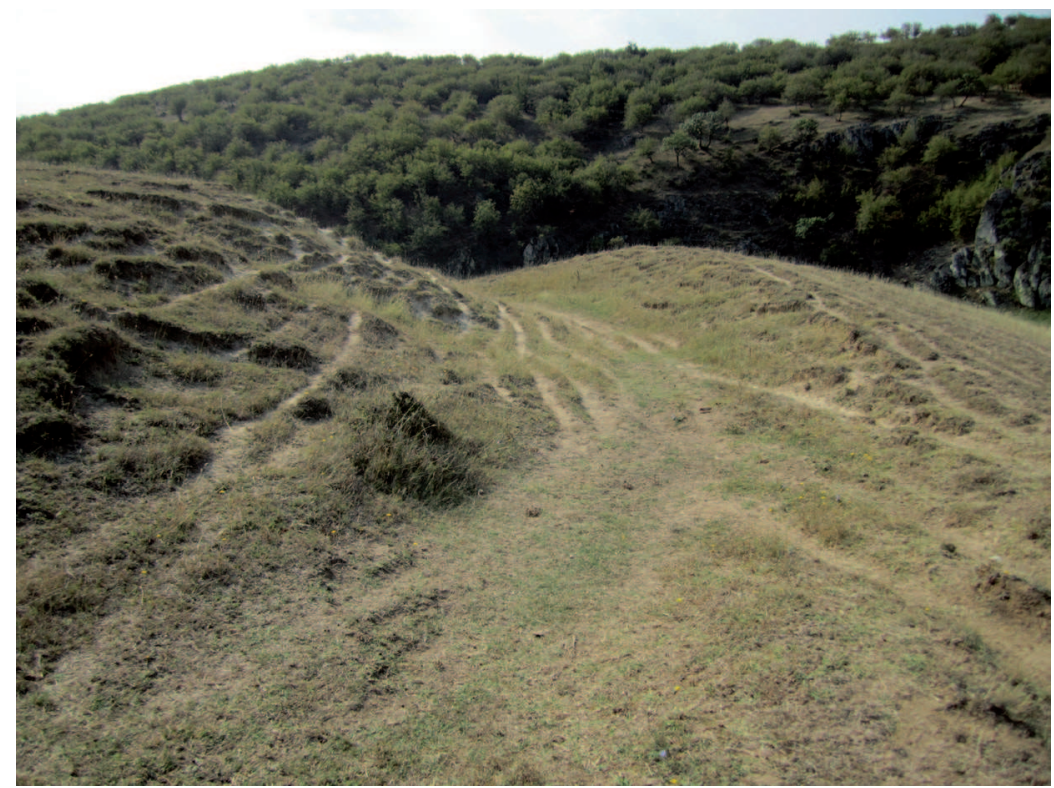

Fig. 3. Route ancienne des mines d'Altin Tepe à Beidaud. Cl. A. Comfort.

plus fine de table. L'ouverture de deux sections, au N. et l'O., n'a pas permis d'identifier la présence claire d'annexes. Ces travaux se sont étendus par la suite à un troisième édifice, connu sous le nom de « Maison du Pêcheur » et partiellement étudié en 2006 par V. Lungu. Disposé o,6 km au N. de « Casa romana I », il constitue à ce jour le seul bâtiment étudié le long de la route septentrionale. Durant ces travaux conduits sur une période très courte, deux murs, d'orientation N.E., ont pu être partiellement dégagés, sans saisir pour autant le plan d'ensemble de cet habitat. ZI, large de $\circ, 8 \circ \mathrm{m}$, est ainsi conservé sur I, $8 \circ \mathrm{m}$ de longueur et dispose encore de deux assises. Un four lui est peutêtre associé.

Sur une toute autre échelle, les travaux de photo-interprétation (A. Comfort, R. Delfieu, M. Guy) se sont poursuivis pour la troisième année consécutive. Ils se sont concentrés essentiellement sur les secteurs proches du golfe de Baia, inscrits dans le triangle Mihai Viteazu-Beidaud-Babadag. Ce dernier semble avoir constitué durant l'Antiquité un carrefour routier important, ainsi qu'un bassin de peuplement dynamique. Plusieurs axes avaient été identifiés en 20II, en particulier deux routes reliant les mines d'Altin Tepe à l'habitat fortifié voisin de Beidaud (fig. 3) et à la cité d'Istros. De même, le tracé de la voie romaine Aegyssus-Tomis avait pu être esquissé, en particulier 
à proximité de la forêt de Babadag, tandis qu'une borne, supposée être en relation avec la route Baia-Slava Russa (anc. Libida), avait été découverte dans le village de Caugagia. Enfin, un gué avait été localisé au S.E. de Baia. L'enquête menée cette année a permis tout d'abord de localiser avec précision le lieu de découverte de la borne de Caugagia, $2 \mathrm{~km}$ au sud de Slava Russa, à l'endroit où le gazoduc croise la route moderne conduisant à Două Cantoane. Cette information confirme ainsi les conclusions avancées en 20II et permet de porter à deux le nombre de bornes milliaires se rapportant à la cité antique de Libida. Le tracé de cet axe de circulation, dont le point de départ n'est autre que Noviodunum, sur le Danube, semble recouper en grande partie la route actuelle, expliquant les difficultés entourant son repérage sur les clichés aériens ou satellite. Son croisement avec l'axe littoral Aegyssus-Tomis s'opérait à la hauteur de Două Cantoane, soit $4,5 \mathrm{~km}$ au N. du village moderne de Baia, dans un secteur qui a conservé jusqu'à nos jours son rôle de carrefour régional. Les vestiges déjà connus d'un vicus d'époque tardo-romaine, présents à l'O. et à l'E. de la route moderne, soulignent en ce sens l'importance de ce croisement qui marque le point de départ de plusieurs axes dont deux au moins se dirigent à l'E. au S.E. en direction de la cité d'Orgamè, le premier en s'engageant au nord des villages modernes de Ceamurlia de Jos et Lunca, le second en suivant la voie littorale. Cette étude a permis d'affiner parallèlement nos connaissances sur l'axe littoral Aegyssus-Tomis. Il semble en effet que, suivant un modèle partagé par le nord de l'Égée, le tracé de cette route régionale se soit affranchi à une certaine époque des anciens établissements grecs littoraux en évitant le village de Mihai Viteazu par où passe également la voie Altin Tepe-Istros. Elle traversait alors un vicus localisé à l'O. du village moderne dont plusieurs formations circulaires, correspondant vraisemblablement à des maisons, sont nettement visibles sur les clichés satellites et aériens. En remontant vers le N., elle passait par l'actuel village de Panduru où un établissement du Haut et du Bas-Empire a été découvert durant cette mission à l'E. et au N.E. du village. Une bande large de $8 \mathrm{~m}$, légèrement bombée, localisée au $S$. d'un gué disposé à l'E. de Panduru, semble lui appartenir. Au N.O. de Baia, cette voie continuait entre deux collines, suivant un tracé parallèle à un chemin moderne, avant de franchir un col où un bloc conservé sous forme fragmentaire $(0,40 \times 0,40 \times 1,00 \mathrm{~m})$ a été découvert. La route ancienne, large de $7 \mathrm{~m}$, se laisse ici facilement appréhender dans le paysage moderne grâce aux talus qui la délimitent sur ses deux côtés. À proximité du carrefour de Două Cantoane, elle se perd en revanche dans la descente, non loin de deux ruisseaux, mais une haie large de $7 \mathrm{~m}$ pourrait matérialiser son tracé. Nous la retrouvons enfin à nouveau un peu plus au N. de la route nationale Baia/Braila, sous la forme d'un alignement de terre pâle qui remonte vers le monastère de Codru et la forêt de Babadag. 
Ces résultats nous ont donc conduits à réaliser parallèlement plusieurs missions de reconnaissance et de diagnostic sur le terrain, lesquelles ont enrichi de façon notable la carte archéologique régionale. Parmi les nouveaux sites enregistrés, deux concernent le triangle Enisala-Orgamè-Cearmulia de Jos et permettent d'éclairer l'occupation du centre de cette vaste péninsule. Une visite du site « camp miliaire », identifié par l'équipe de photo-interprètes en 2010 au sud de la nécropole d'Enisala, s'est soldée ainsi par la découverte de deux monnaies-pointes de flèche et d'un large matériel céramique qui couvre les $\mathrm{VI}^{e}-\mathrm{IV}^{\mathrm{e}}$ s. av. J.-C. De même, un nouvel habitat littoral a été localisé à l'O. du village moderne d'Enisala, dans le creusement d'une large tranchée qui longe au $S$. la route moderne (A. Baralis, V. Lungu).

La plus grande surprise provient toutefois des fouilles de diagnostic conduites du 4 août au Ir septembre (A. Baralis, V. Lungu) sur le site qui occupe le littoral nord de la péninsule de Iancina, au lieu-dit Călugăra. Distant de 10,4 km à vol d'oiseau de la cité d'Orgamè, cet établissement se présente sous la forme d'un vaste plateau de marne claire, de $0,34 \mathrm{~km}(\mathrm{~N} . / \mathrm{S}$.) sur $0,32 \mathrm{~km}$ (E./O.), recouvert par une végétation steppique. Il se prolonge plus au sud dans un champ cultivé sans que ses limites méridionales puissent être appréhendées. Un premier vallon, long de $0,08 \mathrm{~km}$, le sépare au S.E. d'un étroit plateau qui lui fait face, tandis qu'une semblable formation se retrouve à l'O. du site. Les ramassages de surface éclairent une occupation continue de cet espace, depuis le Hallstatt jusqu'au III ${ }^{\mathrm{e}}$ s. apr. J.-C., suivie d'une dernière phase durant la période médiévale. Quelques fragments de l'Âge du Bronze et du Chalcolithique complètent l'éventail chronologique auquel s'ajoutent le long des rivages orientaux les traces d'une occupation ottomane, marquée par une couche épaisse de cendres et de charbons, contenant de nombreux ossements d'animaux et de vertèbres de poissons, observable en profil. Autre élément frappant, la superficie globale de cet habitat dépasse les io ha, soit une surface trois fois supérieure à l'espace urbain intra-muros d'Orgamè/Argamum, tout du moins telle que reconnue pour l'époque tardo-romaine. Il semblait dès lors légitime de s'interroger sur la nature de l'établissement de Călugăra, ainsi que sur la place qui fut la sienne au sein d'un réseau d'occupation spatiale marqué jusqu'ici par la densité des implantations coloniales grecques le long de la côte (Orgamè, Baia 2, Baia 3-Caraburun, CaraburunAtchik Suhat). Dans cette perspective, quatre secteurs de fouilles ont été ouverts, installés aux extrémités E. (secteur A) et S.E. (secteur B) du plateau, ainsi qu'au S.O. (secteur C) et au N.O. (secteur D) du site. La phase la plus ancienne identifiée durant ces travaux n'est autre que le Premier Âge du Fer, présent en secteurs A et D. Dans ce dernier, deux niveaux de sol ont été observés. Le premier ne conservait dans les limites du sondage que trois trous de poteaux, tandis que le second a livré deux fosses dont l'une (diamètre 
de $0,6 \mathrm{I} \mathrm{m}$ ), engagée partiellement dans le profil, était bordée dans sa moitié E. de cinq trous de poteaux. De même, en secteur A-C 3 (carrés II-I2), un édifice quadrangulaire, large de 2,67 m, a pu être partiellement saisi. Il reposait sur des murs en adobes larges de $0,37 \mathrm{~m}$. Ensemble, ils confirment l'occupation contemporaine d'une grande partie du plateau, éclairant dès cette époque l'existence d'un établissement extensif au sein de la carte archéologique régionale. La vie sur ce site semble se poursuivre sans interruption après la fondation de la colonie grecque voisine d'Orgamè. Deux édifices de la fin du VI et du premier quart du $\mathrm{V}^{\mathrm{e}} \mathrm{s}$. av. J.-C. ont été partiellement dégagés en secteur $\mathrm{A}$ (sections $\mathrm{C}_{2}$ et $\mathrm{C}_{3}$ ). Élevés eux-aussi en adobe, suivant un plan et une orientation N./S. similaires, ils étaient accompagnés d'un mobilier important dominé par la céramique modelée de tradition Babadag, associée à une série de fusaïoles et de pesons, auxquels s'ajoutent plusieurs monnaies-pointes de flèches, quelques fragments de céramique corinthienne ou à figures noires et des amphores de divers centres producteurs (Lesbos, Chios, Thasos). Les couches supérieures, souvent perturbées par les labours, ont livré pour leur part des fragments d'adobes ou de clayonnage, des fragments d'amphores majoritairement de Thasos et de la céramique fine à vernis noir, des pointes de flèche de type scythe et quelques tuiles corinthiennes qui attestent de l'adoption progressive de techniques de construction grecques. De cette période, on note la présence de plusieurs fosses disposées en marge de l'habitat, en secteur B. Leur remplissage contenait notamment une quantité notable de graines de blé carbonisées, plusieurs éléments d'adobe, de nombreux fragments d'amphores et de vases modelés, ainsi que des vertèbres de poisson et deux têtes de chiens pour lesquelles il est encore prématuré de conclure si leur déposition relève d'un acte rituel ou d'une consommation. La question des limites de cet habitat se pose naturellement et s'avère directement connectée à l'existence supposée d'une levée de terre présente aux marges de l'établissement, laquelle aurait été destinée à supporter une éventuelle palissade. Conformément aux données issues des ramassages de surface, une maison du II $s$. apr. J.-C., mise au jour en secteur A-CI, rappelle la continuité d'occupation à Călugăra jusqu'à la fin du Haut-Empire. Élevé lui-aussi en terre crue, cet édifice a beaucoup souffert des labours qui n'ont conservé en place que son seuil en pierres sèches et le dormant de la porte. Après six siècles d'interruption, la vie reprend une fois de plus à Călugăra comme l'illustre la maison médiévale étudiée en secteur C (fig. 4). Accompagné d'un matériel de type Dridu des IX $\mathrm{X}^{\mathrm{e}}-\mathrm{X}^{\mathrm{e}} \mathrm{s}$. apr. J.-C., ce bâtiment unicaméral de 5,42 X3,88 m est installé à la limite ouest du site, en surplomb du vallum. Il est construit sur soubassement de pierres sèches avec élévation en adobes et couverture de tuiles. Un foyer ponctue près de la paroi N.O. un sol en terre battue. Enfin, les reconnaissances menées sur la nécropole tumulaire qui semble entourer cet établissement ne se sont pas avérées concluantes. Les anomalies 


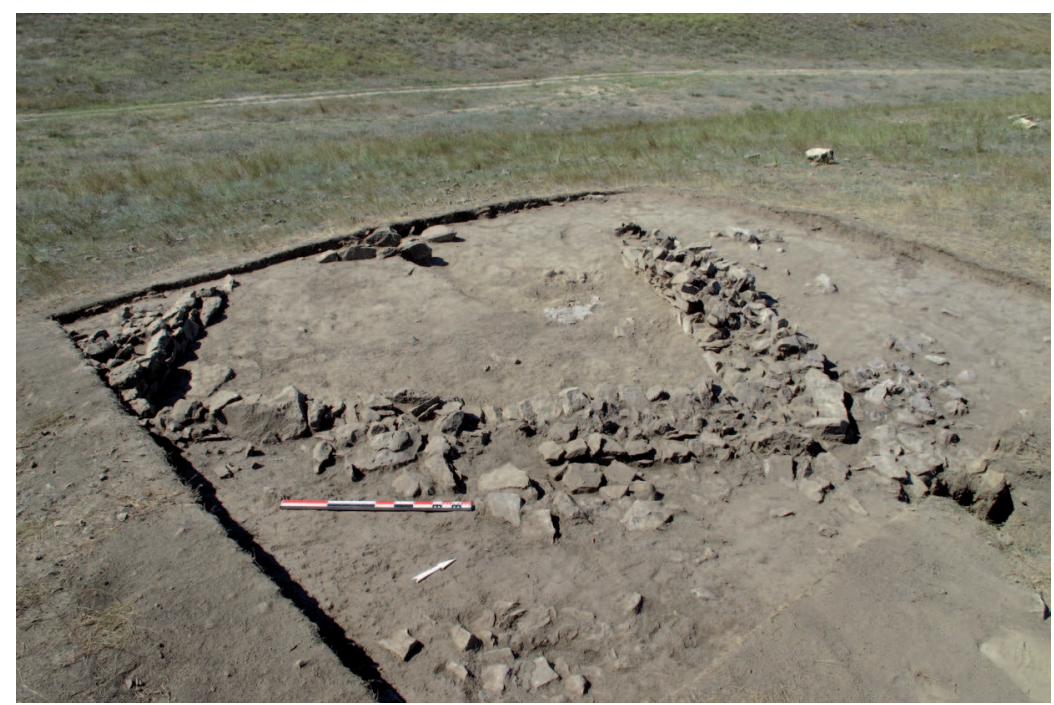

Fig. 4. Călugără, édifice médiéval, secteur C. Cl. A. Baralis (CCJ).

présentes au S.O. du site ont livré un matériel céramique essentiellement médiéval. La fouille d'un des tumuli disposés sur le plateau qui surplombe au S.E. le littoral n'a livré pour sa part aucun contexte. Les recherches menées cette année à Călugăra démontrent donc l'existence d'un site majeur, inscrit dans un contexte gète, établi sur le littoral. Ses dimensions remarquables étonnent car son fonctionnement prend place à un moment où se développent non loin de là, plus au $S$., la colonie d'Orgamè, ainsi que les sites grecs étudiés autour du golfe de Baia, éclairant sous un jour nouveau la question des territoires, tout comme celle des relations que nouent colons et éléments de population locale. Par sa présence, Călugăra confirme toutefois à son tour le caractère modeste du territoire d'Orgamè, tout du moins durant les deux premiers siècles de son existence, au sein d'un contexte régional particulièrement complexe dans lequel les établissements grecs exercent un indéniable pouvoir d'attraction, comme le rappellent le développement à ses marges des sites de Zimbru et de Vişina.

Les résultats obtenus par la mission franco-roumaine ont été présentés du i8 mai au Is juin 2012 à Bucarest, au Musée du paysan roumain, dans le cadre de l'exposition «Dobrogea, entre terre et mer, l'empreinte du temps et des hommes », organisée par J.-P. St Martin sous les auspices du MAEE, de l'Ambassade de France et de l'Institut culturel français de Bucarest. Ces installations sont actuellement visibles à l'ICEM-Tulcea. 


\section{Apollonia du Pont, Sozopol, dpt. de Bourgas, Bulgarie}

Plusieurs campagnes d'analyse et d'étude du matériel ont ponctué, tout comme à Orgamè, l'année 2012. Le programme archéométrique conduit par $\mathrm{P}$. Dupont a ainsi permis d'échantillonner en mars, grâce à la collaboration active de nos confrères (A. Boshkova, M. Gyuzelev, A. Mintchev, M. Tonkova), l'intégralité des grands sites d'occupation grecque du littoral bulgare, portant à près de 129 fragments le total des spécimens analysés depuis le début du programme en 20ro. Leur répartition géographique observe la ventilation suivante : Apollonia du Pont (86), Mésambria (I5), Anchialos (4), Sladkite Kladentsi (Bourgas) (2), Odessos (5), Dionysopolis (3), Bizoné (3) auxquels s'ajoutent 8 fragments provenant d'Héraclée du Pont. Les mesures ont été effectuées au Laboratoire d'archéométrie de la MOM-Lyon en spectrométrie de fluorescence X sur 17 éléments majeurs ( 8 majeurs + traces). Les résultats des productions à vernis noir ont été confrontés avec des références attiques et de Grèce de l'Est, notamment de Pergame et Candarlı; les amphores de type héracléote avec quelques tessons provenant d'Héraclée même et quelques autres spécimens d'Orgamè. Concernant Apollonia du Pont, plusieurs groupes géochimiques distincts, locaux ou régionaux, ont été mis en évidence, à commencer par un ensemble vraisemblablement local rassemblant des productions de céramique commune à pâte grise et claire. Ce dernier intègre également un fragment de la sole du four de potier découvert dans le secteur « Iujna krepostna stena », ainsi qu'un fragment de tuile provenant du temple le plus ancien fouillé sur l'île de St Cyriaque et reconnu comme celui d'Apollon Ietros. Un second groupe recouvre des vases à vernis noir issus essentiellement de la nécropole de Kalfata. À une étape préliminaire du travail, en 20I0, il avait déjà été observé une nette différence de composition avec les références attiques et grecques orientales, excepté peut-être une vague ressemblance de composition avec certaines références de Samos et d'Éphèse, mais il existe aujourd'hui une forte suspicion en faveur de leur origine locale ou loco-régionale. Celle-ci ne pourra toutefois être validée que lorsqu'on aura établi parallèlement une ressemblance de composition avec des références locales indubitables et écarté aussi le risque d'une importation de Thrace égéenne notamment, l'étude archéométrique de F. Blondé et $\mathrm{M}$. Picon sur ces régions étant obérée par des lacunes d'échantillonnage sur certains sites, notamment celui d'Ainos ouvrant vers la Thrace pontique par la vallée de l'Hèbre. On notera d'ailleurs, non sans intérêt, qu'un fragment de lécythe polychrome intègre cette famille, ouvrant la possibilité d'une production locale d'une partie de ces vases particulièrement répandus à Apollonia. À ce stade, la répartition des vases à vernis noir traduit encore une nette prépondérance des importations attiques à Apollonia (20 spécimens assurés et to très probables) par rapport aux 
vases de production locale (I4 spécimens plus I polychrome), mais ce résultat doit être nuancé sur le plan chronologique car les productions locales relèvent majoritairement du seul IV ${ }^{e}$ s., alors que les importations concernent une période bien plus large incluant également le $\mathrm{V}^{\mathrm{e}}$, bien présent dans la ville. Un troisième groupe recouvre enfin les diverses tuiles timbrées mises au jour dans la nécropole, ainsi que sur les trois ensembles d'édifices de Messarite. Concernant Mésambria, les productions propres à cette cité incluent des cruches à pâte claire découvertes dans le cadre des fouilles de la nécropole (dir. A. Boshkova) auxquelles s'ajoutent deux fragments de canthare de type West Slope de la variante « pontique » et un fish-plate à pâte grise découvert à Apollonia dans le secteur urbain UPI-XI-XII 5I5, éclairant très partiellement les échanges entre ces deux cités. Par ailleurs, un groupe marginal correspond à des importations de vernis noir de Callatis, auquel s'ajoutent deux spécimens de céramique commune respectivement à pâte grise et claire, diffusés à Bizonè et Odessos. Des analyses complémentaires sont en revanche nécessaires pour les fragments provenant d'Héraclée ou reconnus comme tels, ainsi que pour les vases supposés d'origine ionienne.

Parallèlement, plusieurs campagnes d'études ont réuni à Sozopol T. Blanco, L. Claquin, M. Damyanov et A. Riapov autour de la céramique incluse dans les trois volumes monographiques actuellement en cours de préparation. Ces travaux ont concerné respectivement le matériel provenant des secteurs fouillés en 2005-2006 dans la nécropole de Kalfata (M. Damyanov, L. Claquin), du secteur urbain UPI-XI-XII 5 Is étudié en 2007-2008 (T. Blanco, L. Claquin, A. Riapov), ainsi que des édifices ruraux de Messarite (L. Claquin, A. Riapov, dessins T. Bogdanova). L'analyse du matériel de la nécropole permet ainsi de situer avec précision l'intégralité des contextes rituels (dépôts et foyers) associés aux enclos funéraires présents à l'E. de la voie littorale, face au secteur franco-bulgare fouillé en 2002-2004. Elle conforte la chronologie avancée dans le premier volume monographique ${ }^{2}$, tout en replaçant la construction des plus anciens périboles sur cet espace exposé aux embruns marins dans le second quart du $\mathrm{IV}^{\mathrm{c}}$ s. av. J.-C. ${ }^{3}$. Ces résultats ont fait l'objet en ce sens d'une présentation le 5 octobre 2012 par M. Damyanov lors du colloque international organisé par le Musée archéologique de Nessebar « Ancient Greek necropolises along the Black Sea Coast ». Les

2 A. Hermary, K. Panayotova, A. Baralis, A. Riapov, M. Damyanov, Apollonia du Pont (Sozopol). La nécropole de Kalfata ( $V^{e}$-III' s. av. J.-C.). Fouilles franco-bulgares (2002-2004), Bibliothèque d'Archéologie Méditerranéenne et Africaine n ${ }^{\circ}$ 5, Paris-Aix-en-Provence, 2010, $300 \mathrm{p}$.

3 A. Baralis, K. Panayotova, « Burial enclosures and spatial organization of the Classical and Early Hellenistic Necropoleis of Apollonia Pontica, Kalfata/Budjaka area », in K. Sporn (éd.), Griechische Grabbezirke klassischer Zeit, Normen und Regionalismen, Mitteilungen des Deutschen Archäologischen Instituts, Athenische Abteilung, sous presse. 
travaux sur la parcelle urbaine UPI-XI-XII 5 I5 offrent de même quelques éclairages intéressants et inédits sur les contextes domestiques d'Apollonia (fig. 5). Depuis l'époque archaïque jusqu'à la fin de l'Antiquité, les vases liés à la cuisine représentent un peu plus de $20 \%$ de l'ensemble des céramiques (plus de 3200 fragments inventoriés); plus de la moitié concerne la préparation des repas, et environ un tiers la cuisson. Pour l'Antiquité grecque, les céramiques de stockage (pithoi) comptent un peu moins de vingt individus différents. La préparation des mets se faisait dans des jattes, mortiers, cratères, lékanès et bols. Ces derniers sont par ailleurs surabondants par rapport aux autres formes de préparation. Notons la présence d'une pierre, ronde, légèrement aplatie, qui aurait pu servir de pilon. Concernant les vases culinaires, les attestations de chytrai sont largement majoritaires tandis que les autres formes culinaires (caccabè, lopas et tagenon) sont faiblement représentées. En revanche, les céramiques non tournées, principalement des pots et quelques jattes, sont assez rares (vingt individus environ). Pour ce qui est des dispositifs de cuisson, au moins deux fours domestiques sont clairement attestés dans le cadre étroit de la parcelle UPI-XI-XII 5 I5 (présence d'évents sur la panse et fortes traces de brûlé sur les parois). Par ailleurs, deux céramiques, de forme circulaire et de grandes dimensions, pourraient être assimilées à des plaques-foyers, bien qu'aucune trace de feu ne soit visible sur ces éléments très fragmentés. Les supports mobiles sont presque absents alors qu'ils sont assez bien attestés sur d'autres sites du monde grec colonial : un seul exemplaire de brasero a été découvert ainsi qu'un fragment s'apparentant à un gril. Les remblais de la fin de l'Antiquité qui s'installent contre la fortification protobyzantine offrent pour leur part un tout autre éclairage sur les relations commerciales qu'Apollonia-Sozopolis entretient entre le $V^{c}$ et le VII ${ }^{\mathrm{e}}$ s. apr. J.-C. Certes, le corpus est comparativement moindre que pour les périodes plus anciennes, mais il n'en demeure pas moins important, puisqu'un peu plus de 450 fragments ont reçu un numéro d'inventaire en raison de leur intérêt et leur importance pour ces périodes. Un nombre notable de vases importés concerne la région de Phocée et ce durant plusieurs siècles comme en témoignent les différentes formes de sigillée Phocéenne tardive ( $L R C$ ou $P h R S W$ ) dont la production s'échelonne dans le temps. Quelques sigillées Africaines $(A R S)$ témoignent également de relations ponctuelles plus lointaines. Concernant les amphores, les restes de LRAi sont de loin les plus nombreux. Si pour une grande partie, il est difficile aujourd'hui d'évoquer leur provenance, quelques tessons présentent une pâte très similaire aux productions du sud de la Turquie, voire de Chypre. Ces cargaisons provenant du bassin Levantin sont confirmées par la présence d'amphores du type de « Gaza » $\left(L R A_{4}\right)$, qui correspondraient aux productions de la fin du $V^{\mathrm{e}}$ et de la première moitié du VI ${ }^{\mathrm{e}}$ s. apr. J.-C. (Inv. 2110, Inv. 2183). Ces analyses seront complétées 


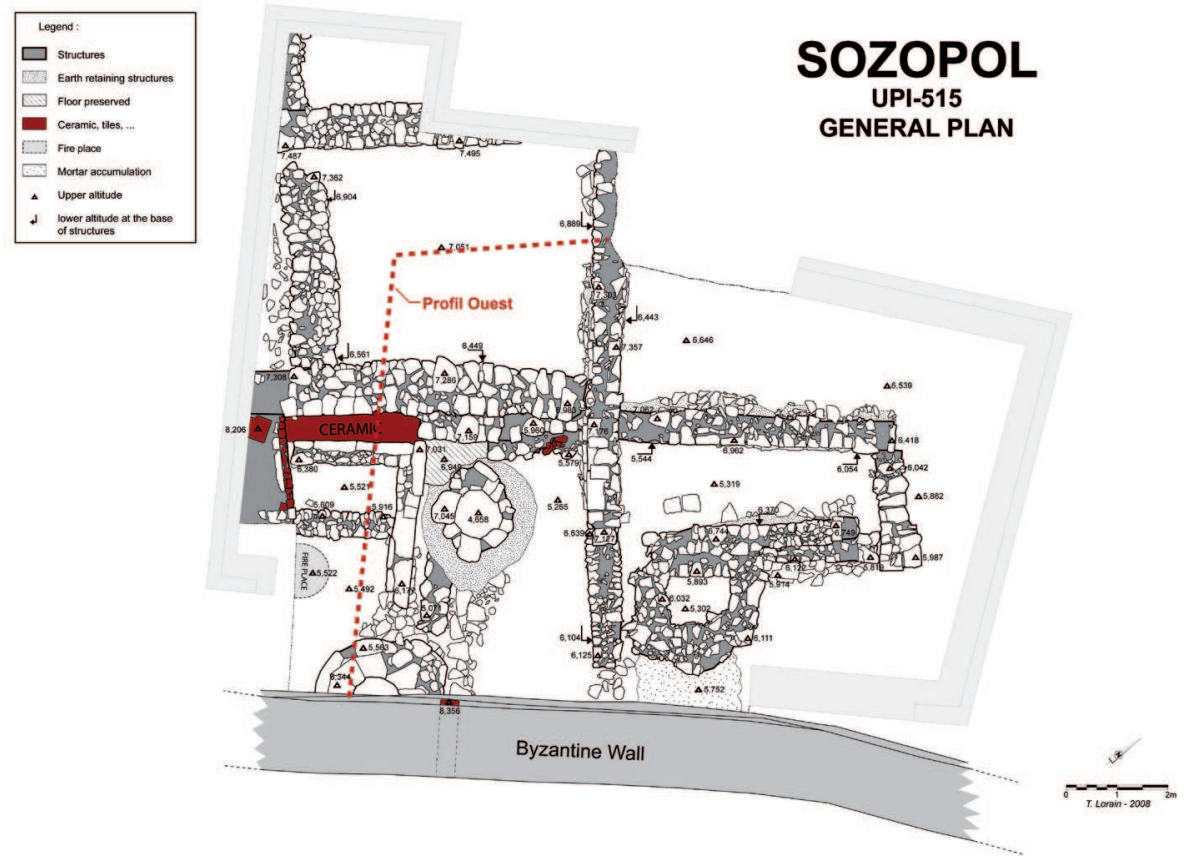

Fig. 5. Apollonia du Pont. Plan des fouilles sur le secteur UPI-XI-XII-515 (T. Lorain).

du 2 au Io novembre par une campagne de relevé photographique du matériel restauré provenant de Kalfata (L. Damelet, A. Damyanov, A. Baralis).

Dans un tout autre domaine, une campagne de carottages a été conduite du 23 mars au 5 avril 2012 (C. Flaux, I. Rossignol, G. Soulet) sur les secteurs littoraux de Gerena et Alepou. Cette opération visait à réaliser les prélèvements nécessaires aux études sédimentologiques (Cerege) et palynologiques (Ecolab) dont l'objectif n'est autre que de fixer, tout comme à Orgamè, l'évolution générale du cadre paléoenvironnemental. Parmi les priorités, notons l'évolution du littoral, l'identification éventuelle des crises sédimentaires induites par les changements dans le réseau d'occupation spatial, tout en esquissant les modifications subies par le couvert végétal. Les carottes obtenues semblent couvrir une grande part de la séquence holocène depuis la submersion de ces secteurs littoraux vers $6000 \mathrm{BP}$ et la stabilisation relative du niveau marin. Les $8 \mathrm{~m}$ de prélèvement obtenus à Alepou illustrent en ce sens l'installation sur l'horizon de base (US E), correspondant au substrat sédimentaire pléistocène récent mis à nu durant la dernière période glaciaire, d'une mince couche de vases très coquillères (US D) laquelle 
témoigne de la transformation progressive de cet ancien espace marin en une lagune fermée, protégée par un cordon sableux, à laquelle appartiennent également les couches US B et $\mathrm{C}$. Cette mission a livré par ailleurs un résultat particulièrement inattendu sous la forme de plusieurs pieux en bois découverts dans trois des carottages à une profondeur comprise entre 5,60 et $6,83 \mathrm{~m}\left(\mathrm{SOZ}_{7}\right.$ à SOZ ter-2) sous le niveau du sol actuel. Ils témoignent de l'existence d'un habitat palafitte encore inédit dont les vestiges sont en cours de datation ${ }^{14} \mathrm{C}$. Le niveau de sol qui leur est associé (US C-I) a livré, dans les limites étroites des carottes, un matériel macrobiologique et paléozoologique particulièrement abondant (charbons, bois, coquillages, arêtes de poisson, ossements d'animaux, graines). Il complète ainsi la longue liste des habitats submergés enregistrés pour les périodes protohistoriques entre l'embouchure du fleuve Ropotamo et le port moderne de Sozopol. Un second gisement a par ailleurs été identifié sur le secteur littoral de Sv. Toma, où un paléosol contenant de nombreux fragments de céramique chalcolithique (identification D. Nedev, K. Leshtakov) a été mis à nu par les tempêtes hivernales. Il comportait I2 sillons parallèles (longueur : I,60 à $4 \mathrm{~m}$; largeur : 0,90 à I m) distants les uns des autres de $0,40 / 0,50 \mathrm{~m}$ et présents sur un espace long de $32 \mathrm{~m}$.

Ces découvertes complètent ainsi un programme archéologique particulièrement soutenu. Une mission d'expertise (J. Thiriot, A. Baralis) a tout d'abord été réalisée durant le mois de mars à la demande de nos confrères sur le chantier « Iujna krepostna stena » (Mur de fortification méridional; dir. Ts. Drajeva avec l'aide active de D. Nedev) à l'entrée de l'antique zone urbaine, dans le secteur du jardin maritime qui borde au S.E. la fortification protobyzantine. Ces travaux ont identifié dans cette zone la superposition de plusieurs édifices d'époque ottomane aux abords de l'ancien circuit de fortification, dans un espace qu'encadre au $S$. une église extra-muros médiobyzantine jusqu'à présent inconnue. Présentant deux étapes distinctes dans sa construction, elle succède elle-même à une nécropole protobyzantine dont les tombes les plus anciennes ne rassemblent que des sujets immatures, inhumés à proximité immédiate de la cité. Ce niveau repose à son tour sur les vestiges d'une zone artisanale qui a livré jusqu'à présent un four à céramique de dimensions remarquables et trois autres unités à la fois plus modestes et plus anciennes destinées au travail du bronze. Seule cette première structure était visible lors de notre intervention sur place, du 22 au 26 mars 20I2, et a bénéficié d'un enregistrement et d'un échantillonnage complet (fig. 6). Un premier relevé a été effectué par $S$. Vasileva. Le four est creusé dans une vaste couche de remblai archaïque contenant une forte proportion de charbon et de matériel provenant d'ateliers métallurgiques disposés plus en hauteur, sur la pente qui marque les anciens abords de la paléo-île de Skamni. Cet horizon recouvrait lui-même une couche de sable stérile 
qui appartient au vaste tombolo qui relie depuis l'Âge du Bronze Skamni au continent sur lequel cinq tombes appartenant au milieu du $\mathrm{VI}^{\mathrm{e}}$ s. av. J.-C. ont été mises au jour durant le mois de septembre. Le four à céramique, hélas, avait été entièrement nettoyé avant son abandon, nous privant ainsi de tout repère chronologique fiable ; il était en partie rempli par des écoulements pluviaux comportant une céramique homogène datée du second quart du $\mathrm{V}^{\mathrm{e}}$ av. J.-C. La structure présente une forme quasi circulaire de 2,98 x 2,64 m, conservée sur une hauteur maximale de $1,20 \mathrm{~m}$. La sole elle-même

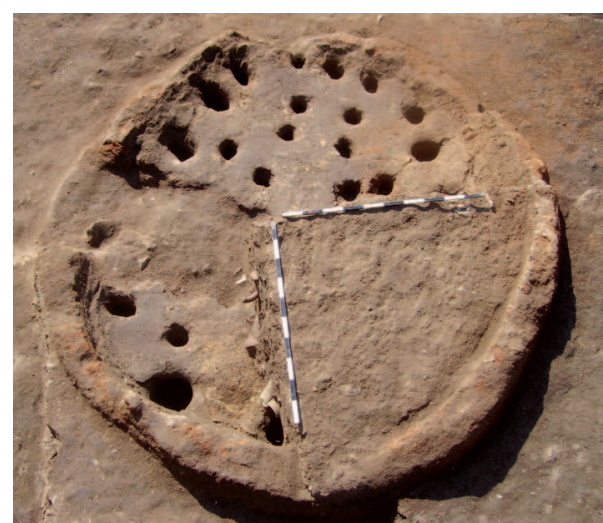

Fig. 6. Apollonia du Pont, secteur « lujna Krepostna stena ». Four à céramique. Cl. D. Nedev.

$(2,60 \times 2,30 \mathrm{~m})$ repose sur un mur axial haut de $0,50 \mathrm{~m}$ et long de $\mathrm{I}, 48 \mathrm{~m}$, bordé de deux couloirs de chauffe et disposé face au foyer. Cette sole est constituée d'un plancher d'argile renforcée d'une armature de barres plano-convexes horizontales (surface plane en haut) s'appuyant sur ce mur et encastrées dans la paroi latérale. Une chambre de cuisson, pratiquement détruite, s'élevait au dessus sur environ 2,50 à $3 \mathrm{~m}$ de haut ; sa porte n'a pas été localisée. La présence d'une voûte de couverture, bien que probable, ne peut pas être arrêtée avec certitude sur la base des vestiges actuellement en place. L'installation d'une sépulture protobyzantine à son extrémité E. a provoqué son démantèlement sur I,IO x $0,60 \mathrm{~m}$. Afin de préciser sa chronologie, nous avons fait appel à une équipe de géomagnéticiens (M. Kovacheva, M. Avramova) de l'Institut de géophysique, géodésie et géographie de l'Académie des sciences de Bulgarie qui a réalisé 38 prélèvements, actuellement en cours d'analyse, sur les trois fours. Ces travaux seront complétés en 2013 par une dernière campagne de relevés destinés à inclure les trois autres fours métallurgiques découverts depuis à proximité.

En parallèle, conformément au projet déposé auprès du MAEE dans le cadre du programme quadriennal, un nouvel axe de recherche a été ouvert, consacré aux nombreux tumuli qui ponctuent le territoire d'Apollonia. $S$ 'ajoutant à leur enregistrement par la numérisation en trois dimensions du terrain par Laser embarqué (Lidar) dont une nouvelle campagne se tiendra en novembre 20I2, une première mission d'étude a été réalisée du 28 mai au $\mathrm{I}^{\text {er }}$ juin 2012 sur celui de Garmitsa. Ce tertre, d'un diamètre de $46,4 \mathrm{~m}$ (N./S.) pour une hauteur de maximale de $7 \mathrm{~m}$, est installé à un 


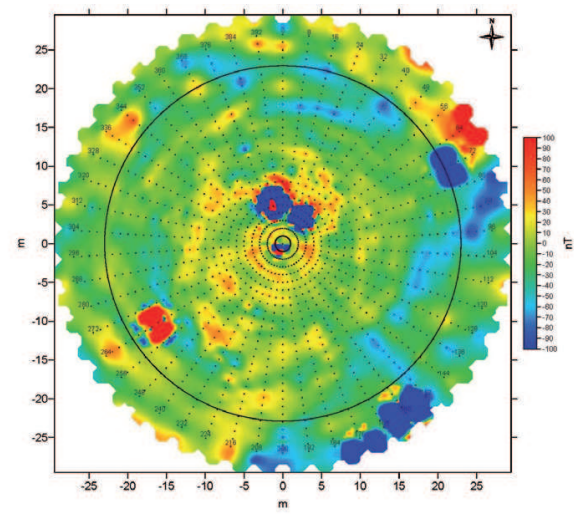

Fig. 7. Relevé géomagnétique du tumulus de Garmitsa (N. Tonkov) kilomètre à l'ouest du village moderne de Chernomorets (anc. St Nicolas), sur une longue crête qui sépare la baie de Gerena au $S$. de celle de Vromos. Ses dimensions le rapprochent d'un autre tumulus de taille imposante préservé dans le secteur voisin de Pachatouria, à proximité immédiate de Chernomorets, tandis que son diamètre dépasse celui du tumulus de Mapite I, lequel correspond vraisemblablement au tertre de Kissir Mikhail Tepe, daté du IV ${ }^{\mathrm{e}} \mathrm{s}$. av. J.-C. et fouillé au début du $\mathrm{XX}^{\mathrm{e}}$ s. par le consul français A. Degrand. Une équipe placée sous la responsabilité de N. Tonkov a réalisé un relevé complet de cette structure en effectuant plusieurs profils électriques, basés sur la mesure de la résistivité du remblai, ainsi que des prospections géomagnétiques (fig. 7). Les résultats obtenus permettent d'écarter dès à présent l'existence de toute structure massive telle qu'une chambre funéraire, un sarcophage ou une ciste, sans que le tracé d'une fosse extensive elle-même n'apparaisse clairement. Ces données orientent dès lors davantage vers des dispositifs plus légers, telles qu'une incinération ou une inhumation primaire, tout en laissant ouverte la présence éventuelle d'un aménagement des abords du tumulus, sous la forme d'un cerclage de pierres ou tout du moins d'une rectification du substrat aux marges du remblai.

En attendant que ces travaux débouchent sur l'ouverture d'un chantier de fouille sur ce tumulus, l'étude du secteur de Messarite s'est poursuivie du 23 avril au I2 mai (A. Baralis, K. Panayotova, T. Bogdanova). La colline de Messarite recouvre le sommet et les versants de la crête disposée au N. du sommet Sv. Ilia. Trois édifices ruraux (Messarite 2, 4 et 6) avaient fait l'objet d'une première campagne de fouilles en 20II, révélant un réseau dense d'occupation durant l'époque classique jusqu'au début de l'époque hellénistique dans cette zone collinéenne relativement proche de la cité. Messarite 2, en position sommitale, avait cependant fortement souffert des différentes interventions modernes dont cette zone a été le théâtre durant le $\mathrm{XX}^{\mathrm{e}} \mathrm{s}$. Ne restait de cet édifice qu'un mur long de 8,I m, bordé immédiatement au $\mathrm{S}$. par un étroit espace occupé par un pithos et un petit muret, lequel abritait deux inhumations primaires de la première moitié du $\mathrm{III}^{\mathrm{c}} \mathrm{s}$. av. J.-C. (SP I et 2). Le démantèlement du foyer présent à l'E. du Mur I a permis d'ajouter à cette liste une troisième inhumation primaire en fosse ( $\mathrm{SP}_{4}, \mathrm{I}, 36 \times 0,70 \times 032$; orientation $\left.5^{\circ} \mathrm{N}\right)$. Les ossements, relativement perturbés, 


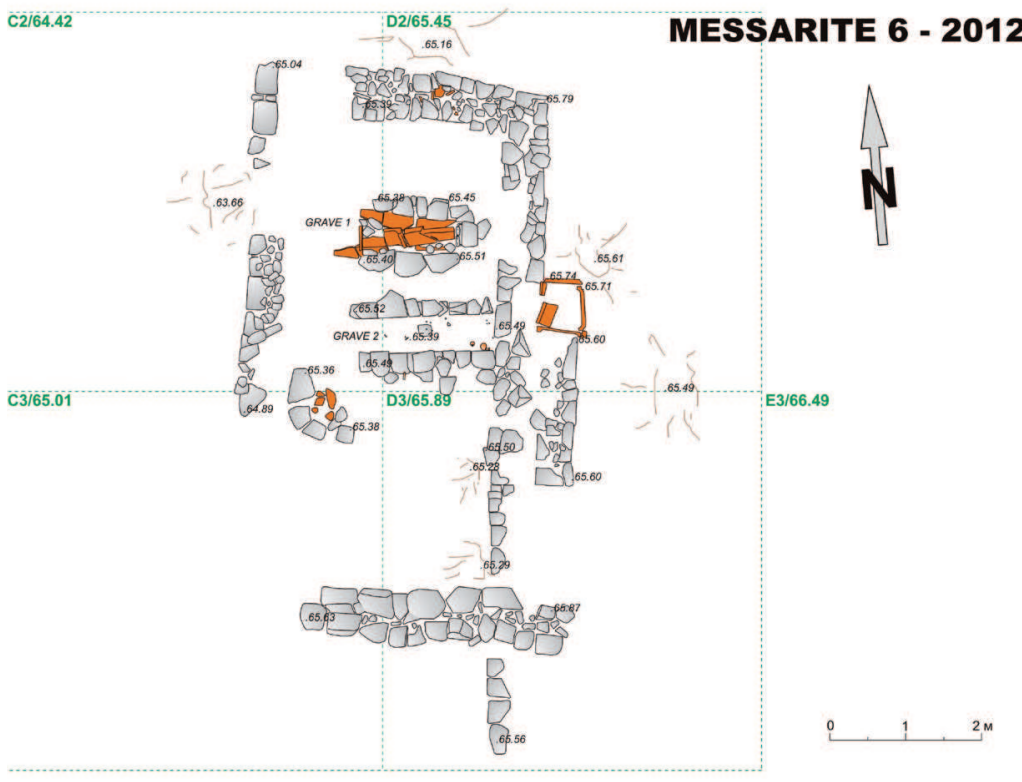

Fig. 8. Messarite 6. Relevé T. Bogdanova, A. Kamenarov.

ne sont que partiellement conservés. Aucun mobilier funéraire n'accompagnait cette tombe dont l'extrémité $N$. de la fosse bénéficiait d'un coffrage soigné en moellons, réutilisé plus tard comme encadrement pour le foyer. Immédiatement au S., une quatrième sépulture ( $\mathrm{SP}_{3}$ ) occupait la zone médiane entre $\mathrm{SP}_{4}$ et $\mathrm{SP}_{\mathrm{I}} / 2$. Deux alignements de blocs doublaient les parois latérales E. et $\mathrm{O}$. de cette fosse, orientée $10^{\circ} \mathrm{N}$, laquelle abritait un sujet adulte placé en décubitus dorsal. Contrairement à SP 4 , un riche mobilier funéraire accompagnait le défunt : une couronne en bronze ornée de perles en terre cuite dorées placée autour du crâne, une fibule miniature en bronze sur l'épaule gauche, deux unguentaria sur la main droite avec un couteau en fer et plusieurs perles en verre, dont certaines étaient également présentes entre les jambes, un miroir en bronze sur la jambe gauche, une « salière » contre le tibia droit à côté d'un pot et deux monnaies en bronze. SP 3 et 4 s'avèrent en l'état contemporaines de SP I et 2.

Cette succession curieuse entre habitat et contextes funéraires se retrouve également sur le site voisin de Messarite 6 (fig. 8) dont l'étude avait débuté elle aussi en 20II. Les travaux ont permis d'achever cette année la fouille de cette structure qui témoigne d'une histoire relativement complexe. La première phase s'articule autour 
d'un espace quadrangulaire de 9,30 x 4,65 m, orienté N./S. Seuls les murs E. et N. sont relativement bien conservés, contrairement au mur occidental dont ne demeurent que quelques segments du parement externe, tandis que le mur S. est, lui, totalement effacé. L'élévation, au-delà des trois premières assises, devait être en briques crues, comme en témoigne la découverte d'une d'entre elles, d'un module de 0,25 x 0,21 x 0,07 m, cuite accidentellement, tandis que la couverture était en tuiles corinthiennes. La construction d'un nouveau mur méridional, qui recoupe le mur E., tout comme le doublement de ce dernier sur 2,60 m, ouvre une nouvelle période dans le fonctionnement de cet édifice. Un coffrage, réalisé en tuiles corinthiennes ( $0,55 \times 0,43 \mathrm{~m})$, est alors placé à l'extrémité N. du nouveau mur O., sans que le remplissage de cette structure ait fourni de matériel, céramique ou macrobiologique. La céramique associée au dernier niveau de circulation (A. Riapov) comprend essentiellement des formes couvrant une large période depuis le second quart du IV ${ }^{\mathrm{e}} \mathrm{s}$. jusqu'au début du $\mathrm{III}^{\mathrm{e}} \mathrm{s}$. av. J.-C., tandis que les nombreuses amphores du $\mathrm{V}^{\mathrm{e}}$ s. découvertes dans le remblai de destruction éclairent la chronologie de la première phase de fonctionnement. Fait marquant, la typologie des amphores recouvre un large éventail et comprend des centres de producteurs à la fois pontiques (Chersonèse taurique, Héraclée du Pont) et égéens (Lesbos, Chios, Mendè, Thasos, Péparéthos, Cnide, Rhodes) avec une forte représentation des conteneurs rattachés au Cercle de Thasos. La troisième étape, qui succède à l'abandon de l'édifice, marque à son tour l'installation de deux sépultures, correspondant toutes deux à une incinération primaire. SP I, au N., disposait d'une couverture de deux rangées de tuiles disposées en bâtière, au sein d'un coffrage en pierres de 0,47 x I,32 m. Le mobilier funéraire comprenait une cruche, deux unguentaria et un strigile en fer. SP 2, distante seulement de 0,27 m disposait elle aussi d'un coffrage en moellons de I,92 x 0,46 m. Son mobilier comprenait deux unguentaria et d'une couronne funéraire en perles de terres cuites dorées. Toutes deux appartiennent au premier quart du III ${ }^{e} s$. av. J.-C.

La surprise principale est venue toutefois du site intermédiaire de Messarite 4 (fig. 9). Une première pièce rectangulaire de 2,10 x 2,10 m (SI) avait été mise au jour en 20II. Adossée immédiatement à l'E. à une seconde plus large $\left(S_{2}, 5,15\right.$ x 3,74 m), elle faisait face à une vaste cour qui se développe plus au S. La campagne de 2012 a permis d'étendre ce secteur de fouilles sur une superficie globale de $350 \mathrm{~m}^{2}$, révélant un complexe rural particulièrement imposant et inattendu dans cette zone située en milieu de pente. Si et $S_{2}$ s'adossent ainsi à deux autres pièces ( $S_{3}$ et 4$)$, disposées au N. Dégagées partiellement, elles ont pu être suivies respectivement sur $3 \times 8 \mathrm{~m}$ et $6,25 \times 8 \mathrm{~m}$. De l'autre côté de la cour, une nouvelle pièce $\left(S_{5}, 2,50 \mathrm{~m}\right.$ de large $)$ s'inscrit dans l'alignement parfait de $S_{\text {I }}$ et permet d'attribuer à cet espace ouvert une largeur de 


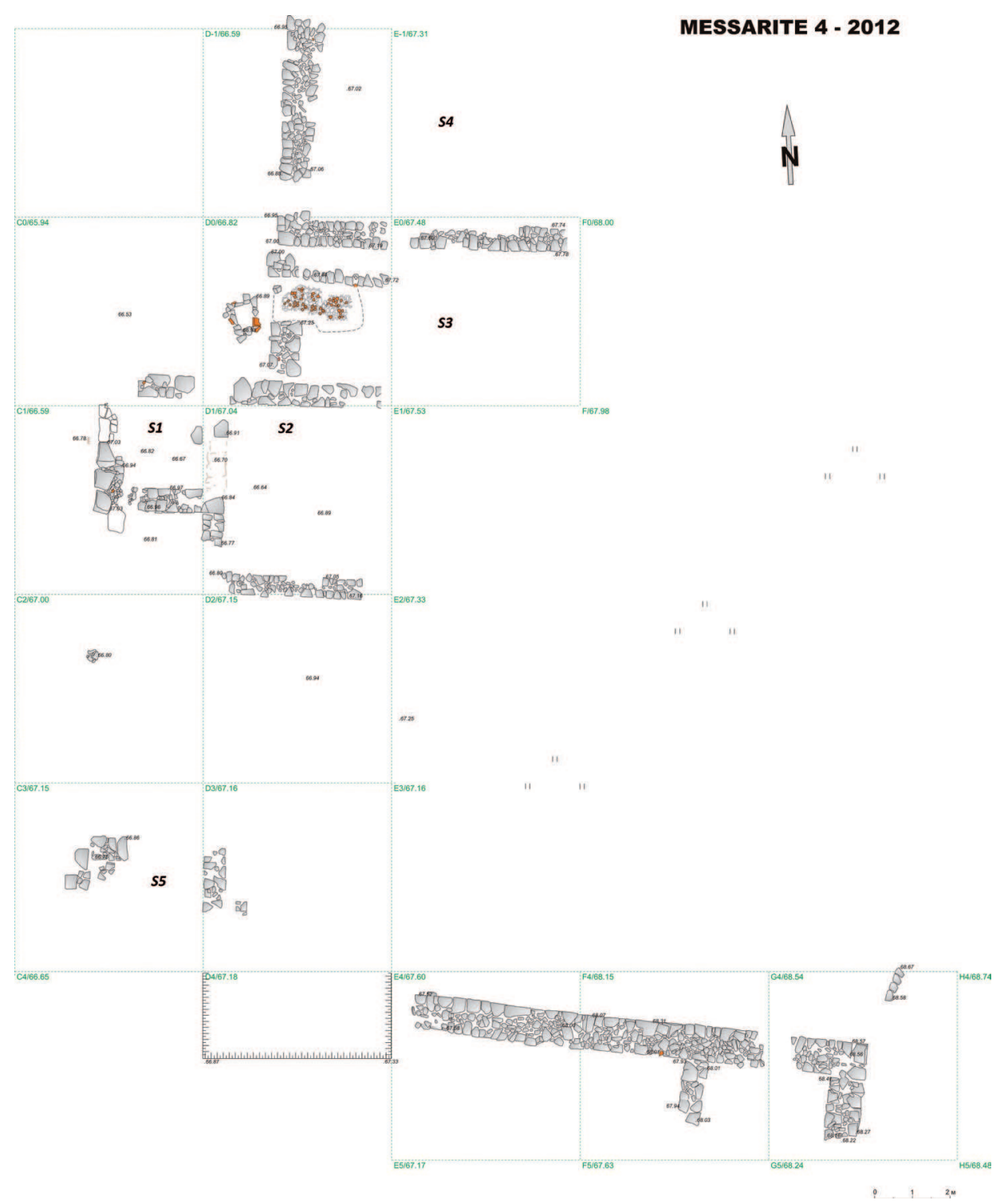

Fig. 9. Messarite 4. Relevé T. Bogdanova, A. Kamenarov.

DHA $38 / 2-2012$ 


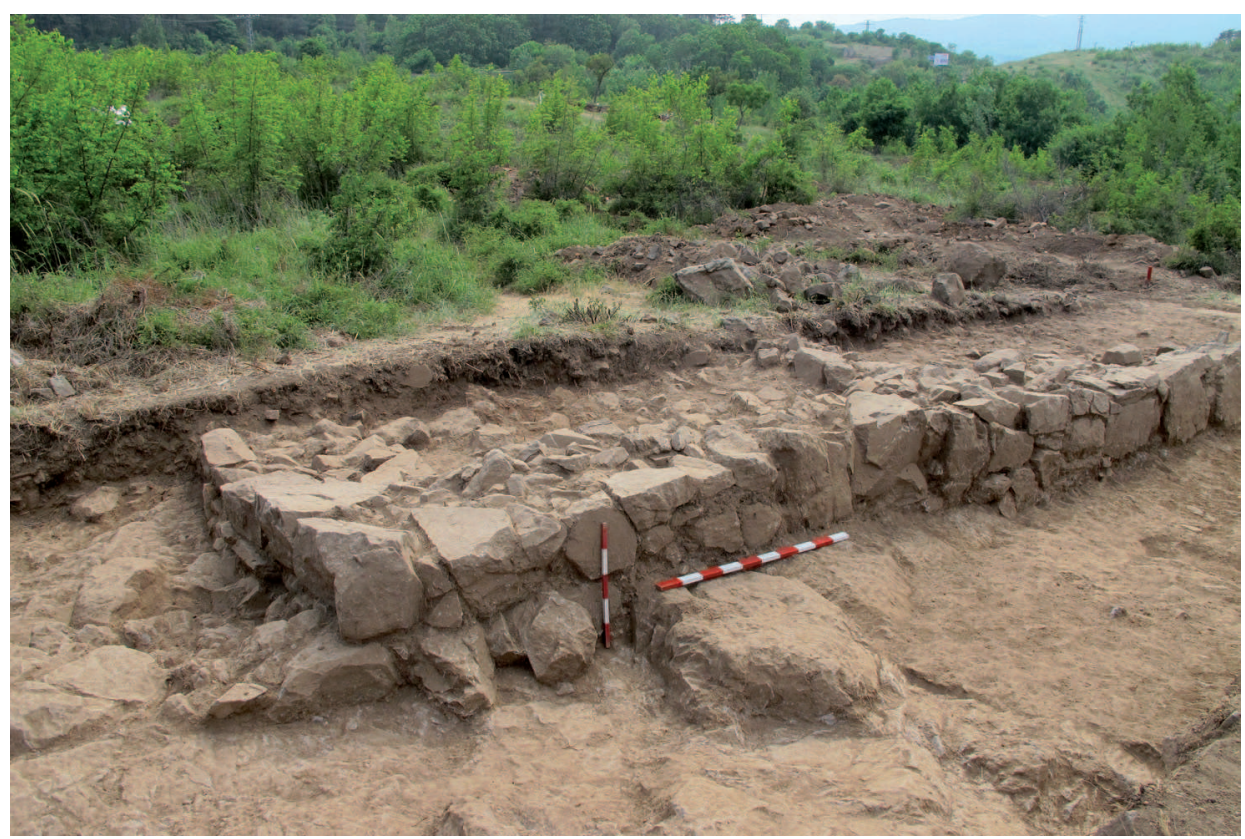

Fig. 10. Messarite 4. Mur E4-G4. Cl. A. Baralis (CCJ).

8,77 m. Si quelques fragments d'amphores appartiennent à la première moitié du Ves. (Lesbos, Chios à col renflé, type protothasien de Zeest), le matériel associé au niveau de circulation de $S_{2}$ et $S_{3}$ relève pour l'essentiel de la fin du $V^{c}$ et du premier quart $\mathrm{du} I \mathrm{IV}^{\mathrm{e}} \mathrm{s}$. av. J.-C. (bolsal, coupe à tige, skyphos et coupe-skyphos). Ces deux pièces sont également les seules à avoir livré de la céramique fine et commune, ainsi que quelques chytrai en association avec une large gamme d'amphores alors que ces dernières constituent l'essentiel du matériel mis au jour dans l'ensemble des pièces qui bordent la cour. Un pied d'amphore de Mendè de la seconde moitié du V $\mathrm{V}^{\mathrm{e}}$ s., conservé dans la maçonnerie d'un des murs, offre d'ailleurs un terminus post quem à la construction de $S_{I}$ et $S_{2}$ dont le niveau de destruction comprend des formes qui s'étendent jusqu'au troisième quart du $\mathrm{IV}^{\mathrm{e}} \mathrm{s}$. Immédiatement au S.E. de ce premier complexe les vestiges d'un second édifice ont pu être identifiés. Le mur N., présent en carrés $\mathrm{E}_{4}-\mathrm{G}_{4}$, a été suivi sur I2,02 $\mathrm{m}$ de longueur. D'une largeur de $0,86 / 0,90 \mathrm{~m}$, il présente un caractère massif et un appareil particulièrement soigné le long de sa façade extérieure, conservée sur deux assises et une hauteur maximale de $0,53 \mathrm{~m}$ (fig. IO). Ce mur forme un angle à l'E. tandis qu'une cloison, d'une épaisseur de 0,61 m, divise l'espace interne de ce large bâtiment. 
Ce dernier accuse également une divergence de $10^{\circ}$ dans son orientation avec les autres murs découverts sur cet étroit plateau ; un décalage qui s'ajoute à une chronologie différenciée car le matériel mis au jour dans le niveau de destruction place le fonctionnement global de cet édifice durant la seconde moitié du IV ${ }^{\mathrm{e}}$ s. av. J.-C., soit à une date légèrement plus tardive. Bien que la relation qu'entretiennent ces deux ensembles ne soit pas encore définie, Messarite 4 illustre une occupation articulée autour de deux groupes de bâtiments particulièrement imposants dont l'étude nécessitera la tenue d'une troisième campagne de fouilles.

Alexandre Baralis (1), Vasilica Lungu (2), Krastina Panayotova (3), Thierry Blanco (4), Guenaelle Bony (5), Anthony Comfort (6), Laurent Clabuin (1), René Delfieu (1), Alexandra Dolea (7), Pierre Dupont (8), Clément Flaux (5), Max Guy (9), Alina Musat (7), Dimitar Nedev (10), Attila Riapov (1), Ingrid Rossignol (11), Ivanka Slavova (12), Marius STReinu (7), Jacques Thiriot (13)

(I) Centre Camille Jullian (UMR 7299), CNRS - Aix-Marseille Université

(2) Institut d'archéologie « V. Pârvan », Institut d'Études Sud-Est Européennes, Bucarest

(3) Institut et Musée archéologique de Sofia, Bulgarie

(4) Université de Bordeaux 3

(5) Cerege (UMR 7330), CNRS - Aix-Marseille Université

(6) Exeter University

(7) Université de Bucarest

(8) Laboratoire d'archéométrie et d'archéologie (UMR 5138), CNRS-MOM, Lyon

(9) Archéoloattes, CNRS (UMR 5I40)

(Io) Musée archéologique de Sozopol

(II) Ecolab (UMR 5245), CNRS - Université Toulouse III

(I2) Université St Clément d'Ochrid - Sofia

(I3) LA 3 M (UMR 7298), CNRS - Aix-Marseille Université 